\title{
Cancer Cell-Membrane Biomimetic Boron Nitride Nanospheres for Targeted Cancer Therapy
}

This article was published in the following Dove Press journal:

International Journal of Nanomedicine

\author{
Shini Feng' \\ Yajing Ren' \\ Hui Li' \\ Yunfei Tang' \\ Jinyu Yan' \\ Zeyuan Shen' \\ Huijie Zhang (D) $^{2}$ \\ Fuxue Chen' \\ 'School of Life Sciences, Shanghai \\ University, Shanghai, 200444, People's \\ Republic of China; ${ }^{2}$ School of \\ Pharmaceutical Sciences, Jiangnan \\ University, Wuxi, 214122, People's \\ Republic of China
}

Purpose: Nanomaterial-based drug-delivery systems allowing for effective targeted delivery of smallmolecule chemodrugs to tumors have revolutionized cancer therapy. Recently, as novel nanomaterials with outstanding physicochemical properties, boron nitride nanospheres (BNs) have emerged as a promising candidate for drug delivery. However, poor dispersity and lack of tumor targeting severely limit further applications. In this study, cancer cellmembrane biomimetic BNs were designed for targeted anticancer drug delivery.

Methods: Cell membrane extracted from HeLa cells (HM) was used to encapsulate BNs by physical extrusion. Doxorubicin (Dox) was loaded onto HM-BNs as a model drug.

Results: The cell-membrane coating endowed the BNs with excellent dispersibility and cytocompatibility. The drug-release profile showed that the Dox@HM-BNs responded to acid pH, resulting in rapid Dox release. Enhanced cellular uptake of Dox@HM-BNs by HeLa cells was revealed because of the homologous targeting of cancer-cell membranes. CCK8 and live/dead assays showed that Dox@HM-BNs had stronger cytotoxicity against HeLa cells, due to self-selective cellular uptake. Finally, antitumor investigation using the HeLa tumor model demonstrated that Dox@HM-BNs possessed much more efficient tumor inhibition than free Dox or Dox@BNs.

Conclusion: These findings indicate that the newly developed HM-BNs are promising as an efficient tumor-selective drug-delivery vehicle for tumor therapy.

Keywords: boron nitride nanospheres, cancer-cell membrane, targeted drug delivery, chemotherapy, biomimetic

\section{Introduction}

Cancer poses a great threat to human health. ${ }^{1}$ Although conventional chemotherapy is one of the standard strategies for cancer treatment, nonselective biodistribution and severe side effects often lead to suboptimal therapeutic outcomes. Following the rapid development of nanotechnology, various types of nanomaterial-based drug-delivery system (DDSs) have been exploited for the treatment of malignant tumors, because DDSs can reach tumor sites through the enhanced permeability and retention effect. ${ }^{2-6}$ Recently, boron nitride nanomaterials have drawn extensive attention from the academic community. ${ }^{7,8}$ As a promising novel inorganic nanomaterial, boron nitride has been applied in many biomedical fields. ${ }^{9,10}$ Boron nitride has a hexagonal structure similar to that of graphene, and possesses remarkable properties of biocompatibility, corrosion resistance, and large thermal neutroncapture cross-sections. ${ }^{11}$ Ciofani et al reported a novel tumor-targeting boron nitride nanotube-based DDS functionalized with folic acid and quantum dots that was able to selectively deliver high doses of boron atoms into tumor cells. ${ }^{12}$ Nakamura et al
Correspondence: Fuxue Chen School of Life Sciences, Shanghai University, Shanghai, 200444, People's Republic of China

$\mathrm{Tel} / \mathrm{Fax}+86-2 \mathrm{I}-6613-5167$

Email chenfuxue@staff.shu.edu.cn
International Journal of Nanomedicine 2021:16 2123-2136

2123 
confirmed the feasibility of using BN nanotubes as boron agent for the boron neutron-capture therapy, a novel binary radiation therapy that can selectively target tumor tissue. ${ }^{13}$ Given their versatility, the applications of hexagonal boron nitride nanomaterials of various shape provide new visions for biomedicine. ${ }^{14,15}$ Among them, boron nitride nanospheres (BNs) with homogeneous spherical structure are considered the most appropriate application in the biomedical field, because of their low structureinduced toxicity. ${ }^{16} \mathrm{Zhi}$ et al reported that BNs can effectively transport DNA into cells, and this delivery system did not affect cell proliferation. ${ }^{17}$ Our research group previously reported that BNs with reasonable surface modification were promising candidates for efficient anticancer drug delivery. ${ }^{18,19}$

Despite boron nitride nanomaterials' excellent properties, major obstacles to their clinical application are poor dispersity, limited blood-circulation time, and lack of tumor selectivity. ${ }^{20}$ The surface hydrophobicity of boron nitride makes it aggregate and quickly cleared from blood by the reticular endothelial system. ${ }^{21}$ Intrinsic characteristics of red blood cells (RBCs), including their superior circulation time and nonimmunogenicity, have been applied to surface modification of DDSs. $^{22,23}$ Very recently, we derived RBC membranes for encapsulation of BNs, which exhibited a monodisperse state in solution, superior circulation half-life, and enhanced stability in a physiological environment. ${ }^{24}$ However, the membrane did not possess targeting moiety and thus could not execute active tumor targeting. Moreover, the tumor specificity of DDS through only passive targeting based on the enhanced permeability and retention effect has not yet been satisfied. Reasonable active-targeting strategies may further improve therapeutic effects and reduce undesirable side effects of chemotherapeutic drugs.

One conventional strategy is to modify DDSs with targeting ligands, including antibodies, peptides, or aptamers. ${ }^{25}$ However, the ligand-mediated targeting strategy is limited by the complex chemical preparation required. ${ }^{26}$ Recently, by utilizing cell-membrane materials for nanoparticle modification, researchers have successfully manufactured DDSs possessing desirable targeting performance. ${ }^{27}$ Examples include natural killer cell membrane-cloaked nanoparticles capable of targeting tumors and producing antitumor immunity, ${ }^{28}$ stem cellderived polydopamine nanoparticles with long-circulating and cancer-targeting capabilities, ${ }^{29}$ and platelet membrane-coated polymeric nanoparticles with the ability to target thrombus sites. ${ }^{30}$ Zhang et al demonstrated that cancer cell membrane-coated nanoparticles developed from homotypic cancer cells can actively target tumor sites. ${ }^{31}$ It can be imagined that through surface decoration with cancer-cell membrane, a promising BN-based DDS capable of specific tumor targeting and immunoescape can be developed.

In this study, a novel cancer cell-membrane camouflage DDS for targeted tumor treatment was developed (Figure 1). BNs were first coated with HeLa cell membrane (HM). Then, doxorubicin (Dox) was used as the chemotherapeutic drug model and loaded on HM-BNs to fabricate the cancer cell-membrane biomimetic BN-based DDS (Dox@HM-BNs). The fabricated Dox@HM-BNs were fully characterized. The Doxloading and $\mathrm{pH}$-dependent drug-release behavior of Dox@HM-BNs was investigated. Afterward, cellular uptake, tumor targeting, and anti-cancer ability of the Dox@HM-BNs were studied.

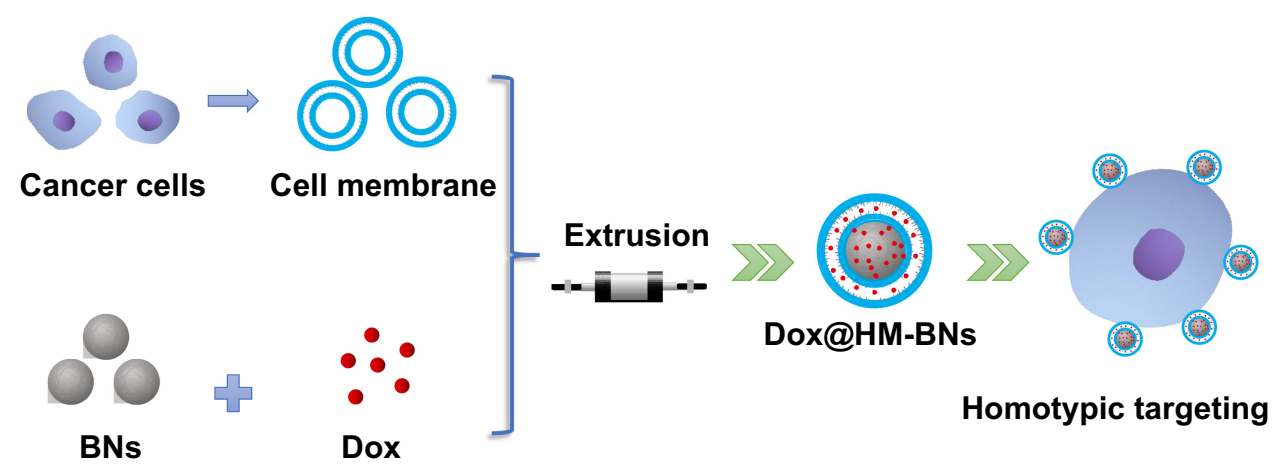

Figure I Schematic of preparation of Dox@HM-BNs and homotypic tumor-targeting drug delivery. 


\section{Methods}

\section{Materials}

Membrane protein-extraction kit, protease-inhibitor cocktail, BCA protein-assay kit, SDS-PAGE gel-preparation kit, and DAPI for nuclear staining were obtained from Beyotime (Haimen, China). A mini-extruder and polycarbonate porous membrane were bought from Avanti Polar Lipids. DMEM, FBS, and trypsin-EDTA were obtained from Gibco Penicillin-streptomycin and a live/dead viability/cytotoxicity kit for mammalian cells were purchased from Invitrogen. The CCK8 assay kit was provided by Dojindo (Kumamoto, Japan). Dox was provided by Sinopharm Chemical Reagent. All other reagents were purchased from Sinopharm Chemical Reagent and used without further treatment.

\section{Cell Culture}

HeLa (human cervical carcinoma cell line), MCF7 (human breast cancer cell line), HEK293 (human embryonic kidney cell line) and J774A.1 (mouse mononuclear macrophage cell line) cells were provided by ATCC. Cells were incubated in DMEM containing 10\% FBS, $100 \mathrm{U} / \mathrm{mL}$ penicillin, and $100 \mathrm{mg} / \mathrm{mL}$ streptomycin. Cells were cultured in an incubator at $37^{\circ} \mathrm{C}$ with a humidified atmosphere. The cell-culture medium was replaced with fresh medium every 2 days.

\section{Isolation of HeLa Cell Membrane}

Isolation of cancer-cell membrane was carried out as per a previous report. ${ }^{31} \mathrm{HeLa}$ cells were detached with trypsin and washed three times with ice-cold PBS. After that, HeLa cells were resuspended in PBS solution. The protease-inhibitor cocktail was used to prevent the degradation of cell-membrane proteins. Cells were disrupted by a Wiggens Homogenizer (Beijing, China) for 20 passes at 30 -second intervals. The homogenized solution of cellmembrane fractions was centrifuged at $3,200 \mathrm{~g}$ for 6 minutes. Collections were then ultracentrifuged at $100,000 \mathrm{~g}$ for another 30 minutes. The transparent HM sediment was collected for subsequent use.

\section{Preparation of HM-BNs}

BNs with uniform spherical structure were firstly fabricated via chemical vapor deposition between $\mathrm{B}(\mathrm{OMe})_{3}$ and ammonia, followed by high-temperature annealing. ${ }^{32}$ The $\mathrm{HM}$ suspension and $\mathrm{BN}$ solution $(1 \mathrm{mg} / \mathrm{mL})$ were mixed at a volume ratio of 1:1. The mixture was added to the mini- extruder for extrusion. Polycarbonate porous membrane with pore diameters of $400 \mathrm{~nm}$ and $200 \mathrm{~nm}$ were used serially. After extrusion for 30 cycles each, the HM-BNs were centrifuged at 13,500 rpm for 12 minutes. HM-BNs were finally collected by freeze-drying. The membrane protein from HM-BNs was quantitatively analyzed with the BCA kit and verified by SDS-PAGE. ${ }^{23}$ Specifically, samples were prepared at a final protein concentration of $1 \mathrm{mg} / \mathrm{mL}$ in loading buffer and heated to $80^{\circ} \mathrm{C}$ for 10 minutes. Samples $(20 \mu \mathrm{L})$ were loaded in each well of a Bio-Rad electrophoresis system. Protein staining was accomplished using Coomassie blue, with destaining in water overnight before imaging.

\section{Dox Loading and In Vitro Release Study}

For drug loading of BNs, $5 \mathrm{mg}$ Dox was added to $5 \mathrm{~mL}$ BN solution at a concentration of $1 \mathrm{mg} / \mathrm{mL}$. The mixture was gently shaken overnight at room temperature, then centrifuged at 13,500 rpm for 20 minutes. The amount of free Dox in the supernatant was detected with microplate spectrophotometry to calculate the amount of Dox loaded onto the carriers. As for the fabrication of Dox@HM-BNs, $5 \mathrm{mg}$ Dox was added to $5 \mathrm{~mL} \mathrm{BN}$ solution at a concentration of $1 \mathrm{mg} / \mathrm{mL}$. The mixture was gently shaken overnight at room temperature. Then, the HM suspension was added to the mixture at a volume ratio of 1:1 with sufficient mixing. Subsequently, the mixture was added to the mini-extruder and extruded for 30 cycles through a polycarbonate porous membrane with pore diameters of $400 \mathrm{~nm}$ and $200 \mathrm{~nm}$. Finally, the product was centrifuged at 13,500 rpm for 12 minutes at $4^{\circ} \mathrm{C}$, yielding Dox@HM-BNs. The amount of free Dox in the supernatant was measured at $480 \mathrm{~nm}$ and the loaded Dox calculated. Drug-loading capacity was calculated as the amount of Dox loading on carrier/the amount of carrier. For in vitro drug-release investigation, 5 mg Dox@BNs and Dox@HM-BNs were dispersed in 10 mL PBS (pH 7.4 and 5.0, respectively). The solutions were kept in the dark with slight shaking. At fixed time points, the supernatant was collected and substituted with equivalent fresh PBS The Dox release was measured with microplate spectrophotometry at $480 \mathrm{~nm}$.

\section{Characterization}

The surface morphology and internal structure of BNs and HM-BNs were visualized with an Emsis Veleta G3 transmission electron microscope at accelerating voltage of $80 \mathrm{kV}$. Energy-dispersive X-ray spectroscopy (EDS) microanalysis 
was performed with scanning electron microscopy (Hitachi SU8020). The $\zeta$-potential, polydispersity index, and average diameter of HM-BNs were determined using a Malvern Nano ZS Zetasizer. Cells with fluorescent staining were visualized with confocal laser-scanning microscopy (CLSM; Occult International LSM710). Flow-cytometry investigation was conducted using a Beckman Coulter MoFlo XDPBD flow cytometer. Chemotherapeutic drug loading and in vitro release profiling were carried out using a Tecan Qenius automatic microplate reader. Boron-atom content in cells and tissue were determined by PerkinElmer Optima 7000DV inductively coupled plasma atomic emission spectroscopy (ICP-AES).

\section{Cell-Viability Assays}

The CCK8 assay kit was used to investigate cell viability after treatment with BNs and HM-BNs. HeLa, MCF7, and HEK293 cells at a density of 4,000 cells/well were inoculated in 96-well plates with $100 \mu \mathrm{L}$ in each well for 24 hours. Then, BNs and HM-BNs at concentrations of $25 \mu \mathrm{g} / \mathrm{mL}, 50 \mu \mathrm{g} / \mathrm{mL}$, and $100 \mu \mathrm{g} / \mathrm{mL}$ were added to the plates, with five replicates for each concentration and incubation continued for 72 hours. Cells treated with PBS was set as control. After that, cells in each well were treated with $10 \mu \mathrm{L}$ CCK8 solution for 3 hours. Absorbance of the culture medium was measured at $450 \mathrm{~nm}$ with an automatic microplate reader and calculated. Cell viability was expressed as the percentage of living cells over untreated cells.

\section{Cellular Uptake}

HeLa and MCF7 cells at a concentration of $5 \times 10^{4}$ cells per dish were inoculated in $1 \mathrm{~mL}$ DMEM at $37^{\circ} \mathrm{C}$ for 24 hours. Subsequently, free Dox, Dox@BNs, or Dox@HMBNs were added to the dish and incubation continued for 4 hours. After that, cells were washed with PBS. Then 200

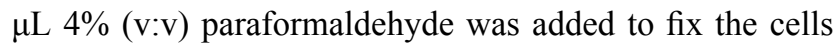
for 10 minutes. After washing twice with PBS, cells were stained with DAPI at room temperature for 20 minutes. Fluorescence was observed with CLSM.

To quantitatively calculate the amount of samples internalized by cells, flow-cytometry analysis was conducted. HeLa cells with a density of $5 \times 10^{5}$ cells/well were incubated in six-well plates for 24 hours. Then, free Dox, Dox@BNs, and Dox@HM-BNs at same concentration (4 $\mu \mathrm{g} / \mathrm{mL})$ were added to the cells. After 4 hours' treatment, cells were collected by trypsin detaching and rinsed with cold PBS. After resuspension in cold PBS, the cellular uptake of Dox@HM-BNs by HeLa cells was investigated by flow cytometry.

Cellular uptake of HM-BNs by HeLa cells and HEK293cells was quantitatively evaluated by ICP-AES. Cells were seeded at a density of $10^{6}$ cells in a $10 \mathrm{~cm}$ petri dish and cultured in DMEM containing 10\% FBS for 24 hours. Then, both cell types were treated with HMBNs at a concentration of $50 \mu \mathrm{g} / \mathrm{mL}$ for 4 hours. After cells had been collected and washed three times, $0.1 \mathrm{~mL}$ $\mathrm{HNO}_{3}(65 \mathrm{wt} \%)$ was added to allow dissolution of the complex overnight. Finally, $9.9 \mathrm{~mL}$ ultrapure water was added to the solution. Boron concentrations in the acidic solutions were measured to quantify the mass of HM-BNs in cells.

The immunoescape study was conducted using the mouse mononuclear macrophage cell line J774A.1. Specifically, J774A.1 cells were seeded in a $10 \mathrm{~cm}$ petri dish at a density of $10^{6}$ cells and cultured in DMEM containing $10 \%$ FBS. After incubation for 24 hours, cells were treated with BNs and HM-BNs at the same BNs concentration $(50 \mu \mathrm{g} / \mathrm{mL})$ for 4 hours. Then, cells were detached by trypsin and washed three times with cold PBS. Subsequently, $0.1 \mathrm{~mL} \mathrm{HNO}_{3}(65 \mathrm{wt} \%)$ was added to the cells and maintained overnight. Finally, $9.9 \mathrm{~mL}$ ultrapure water was added and mixed. Boron concentrations in the solutions were measured by ICP-AES.

\section{In Vitro Antitumor Effects of Dox@HM-BNs}

The cancer cells were seeded at $10^{4}$ cells per well in a 96-well plate at $37^{\circ} \mathrm{C}$ with $5 \% \mathrm{CO}_{2}$. After 24 hours' incubation, $2 \mu \mathrm{g} / \mathrm{mL}$ free Dox, Dox@BNs, and Dox@HM-BNs with the same Dox concentration were added. Five replicates were set for each sample. Subsequently, cells were treated with $10 \mu \mathrm{L}$ CCK8 solution in each well for 3 hours. Absorbance of the culture medium was measured at $450 \mathrm{~nm}$ with an automatic microplate reader and calculated.

Live/dead viability/cytotoxicity assays were also performed. HeLa and MCF7 cells at a concentration of $5 \times 10^{4}$ cells per dish were inoculated in $1 \mathrm{~mL}$ DMEM at $37^{\circ} \mathrm{C}$ for 24 hours. Then, cells were treated with $5 \mu \mathrm{g} / \mathrm{mL}$ free Dox, Dox@BNs, and Dox@HM-BNs with the same Dox concentration for another 12 hours. Subsequently, cells were rinsed with cold PBS and stained with calcein AM and propidium iodide. Finally, fluorescence was identified 
using CLSM. Green fluorescence representedlive cells and red fluorescence dead cells.

\section{In Vivo Biodistribution of HM-BNs}

Female BALB/c nude mice (18-20 g) were used as animal models. Animal studies were approved by the Ethics Committee of Shanghai University and conducted strictly according to the institutional animal care and use program of experimental animals of Shanghai University (approval ECSHU-2020-030). A subcutaneous xenograft tumor model was established by injecting HeLa $\left(5 \times 10^{7}\right)$ cells subcutaneously into mice. After tumor volume had reached $100 \mathrm{~mm}^{3}$, mice were intravenously injected with BNs or HM-BNs (six mice/group) at a BN dose of $10 \mathrm{mg} / \mathrm{kg}$. Animals were killed at 24 hours postinjection. Heart, liver, spleen, lung, kidney, and tumor-tissue samples were collected and lysed in a mixture of $\mathrm{HClO}_{4}$ and $\mathrm{HNO}_{3}\left(\mathrm{HClO}_{4}\right.$ $\left.: \mathrm{HNO}_{3}=1: 9, \mathrm{v}: \mathrm{v}\right)$. The number of boron atoms accumulated in tissue was measured by ICP-AES. The distribution of BNs or HM-BNs in different tissue samples was recorded as percentage of injected dose per gram of tissue.

\section{In Vivo Tumor-Inhibition Effects of Dox@HM-BNs}

In vivo tumor-inhibition treatment started when tumor volume in female Balb/c nude mice had reached $100 \mathrm{~mm}^{3}$ at day 0 . Mice were randomly divided into four groups $(n=6)$. Mice were intravenously injected with PBS, free Dox, Dox@BNs, or Dox@HM-BNs with the same Dox dose $(5 \mathrm{mg} / \mathrm{kg})$. Treatment was conducted every 2 days three times. Body weight and tumor volume were measured and recorded every 3 days for 3 weeks. At 21 days postinjection, mice were killed and tumors collected and photographed. The major organs (heart, liver, spleen, lungs, and kidneys) were excised and fixed with $4 \%$ formalin. Then, organ sections were stained with $\mathrm{H} \& \mathrm{E}$ for evaluation of biosafety. Tumor volume $(\mathrm{V})$ was calculated as $\mathrm{V}=0.5 \times \mathrm{L} \times \mathrm{W}^{2}$ (L, length of tumor; $\mathrm{W}$, width of tumor).

\section{Statistical Analysis}

Student's $t$-test was used for statistical analysis. Data are expressed as means $\pm \mathrm{SD}$. Differences were considered statistically significant at $P<0.05$.

\section{Results and Discussion Preparation and Characterization of HM-BNs}

As shown in Figure 2, it was clearly seen on transmission electron microscopy that BNs had a spherical structure with an average particle size of about $100 \mathrm{~nm}$. The diameter of HM-BNs obtained by extruding BNs with HM was about 120 $\mathrm{nm}$. The enlarged image of HM-BNs showed that the transparent membrane structure of about $10 \mathrm{~nm}$ thickness corresponding to the width of the cell membrane was attached to the surface of the HM-BNs, which indicated that the HM film had successfully wrapped onto BNs after mechanical extrusion. ${ }^{33,34}$ Moreover, those particles were more dispersed compared to pristine BNs without modification. Elemental analysis of HM-BNs was conducted with EDS microanalysis (Table 1). A significant amount of $P$ appeared in the HM-BN sample ( $0.47 \%$ atomic content). Moreover, oxygen and carbon were increased in HM-BN nanocomposites in comparison with BNs. Given that the phospholipid bilayers of cell membrane are fashioned from carbon, nitrogen, oxygen, and phosphorus, this result further indicated successful cellmembrane modification of HM-BNs.

Dynamic light scattering was carried out to determine the average diameter and $\zeta$-potential of HM-BNs. As shown in Figure 3A, BNs had a large hydrodynamic diameter of about $722 \mathrm{~nm}$, due to their surface hydrophobicity and aggregation in solution. In comparison, the hydrodynamic diameter of HM-BNs was down to $138 \mathrm{~nm}$. This suggested
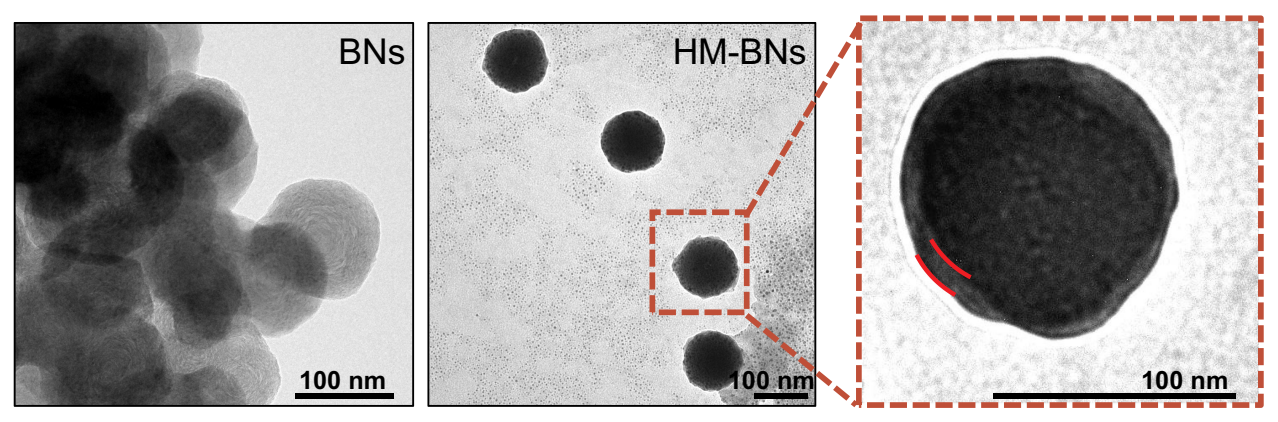

Figure 2 Transmission electron microscopy of BNs and HM-BNs. 
Table I Atomic composition of BNs and HM-BNs analyzed by EDS

\begin{tabular}{|l|l|l|l|l|l|l|}
\hline \multirow{2}{*}{} & \multicolumn{6}{|l|}{ Elements (\%) } \\
\cline { 2 - 7 } & B & N & C & O & P & Total \\
\hline BNs & 31.07 & 31.48 & 14.43 & 23.03 & 0 & 100 \\
HM-BNs & 15.67 & 20.18 & 31.10 & 32.57 & 0.47 & 100 \\
\hline
\end{tabular}

that the HM modification reduced the hydrophobicity of BNs, making it more dispersible in PBS. After being camouflaged with $\mathrm{HM}$, the surface $\zeta$-potential of HM-BNs changed from -34.2 to -14.6 (Figure 3B). This result further demonstrated that BNs had been successfully coated with cell membrane, as the surface charge of HM-BNs got closer to the level of the HM. Then, SDS-PAGE assays were conducted to determine protein profiles on the HM-BNs. The protein composition of HM-BNs was similar to that of $\mathrm{HM}$, indicating that the extrusion process during fabrication had not damaged the membrane proteins on HM-BNs (Figure 3C). Furthermore, the hydrodynamic diameter of the HM-BNs was stable within the testing time period (Figure 3D). Moreover, the polydispersity index of HM-BNs after dispersal in PBS for 14 days was maintained at 0.086 , demonstrating the excellent long-term stability of the HM-BNs (Figure 3E).

\section{Cytotoxicity Assays}

Since a desirable DDS should possess applicable biosafety, cytotoxicity assays of BNs and HM-BNs were performed using the CCK8. HeLa, MCF7 and HEK293 cells were cultured with BNs and HM-BNs at different concentrations for 72 hours. Cancer cells treated with PBS were set as control. As shown in Figure 4, the cell viability of both cancer cells and normal cells remained $>90 \%$ after treatment with HM-BNs for 72 hours within the experimental concentration range, while cells treated with pristine BNs showed slightly lower cell viability at high doses. Our previous research demonstrated the camouflage of RBC membrane improved the biocompatibility of BNs. ${ }^{24}$ From this result, it was concluded that the HM encapsulation reduced the toxicity of BNs, making HM-BNs more compatible for application in the biomedical field.
A

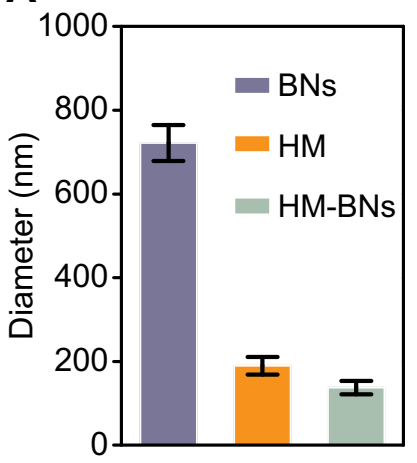

D

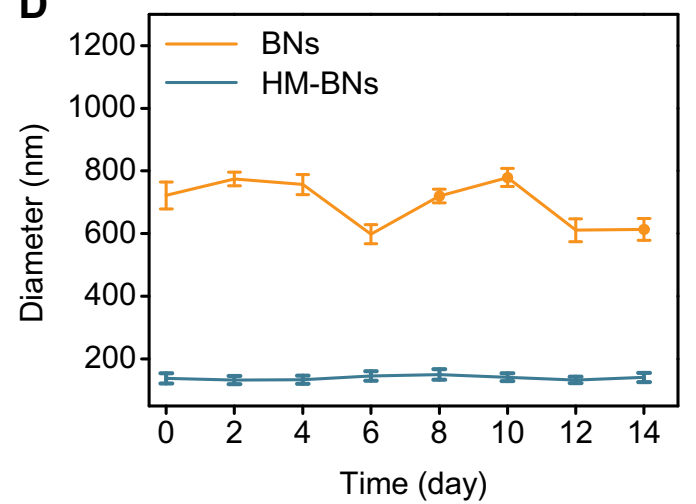

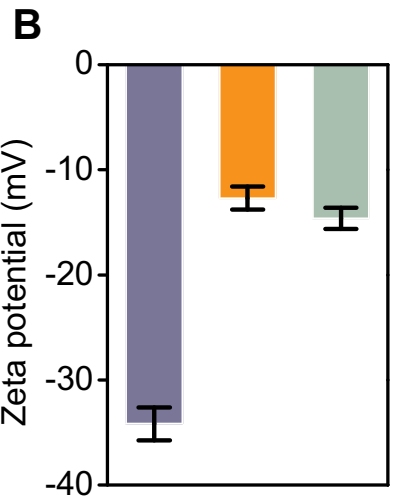
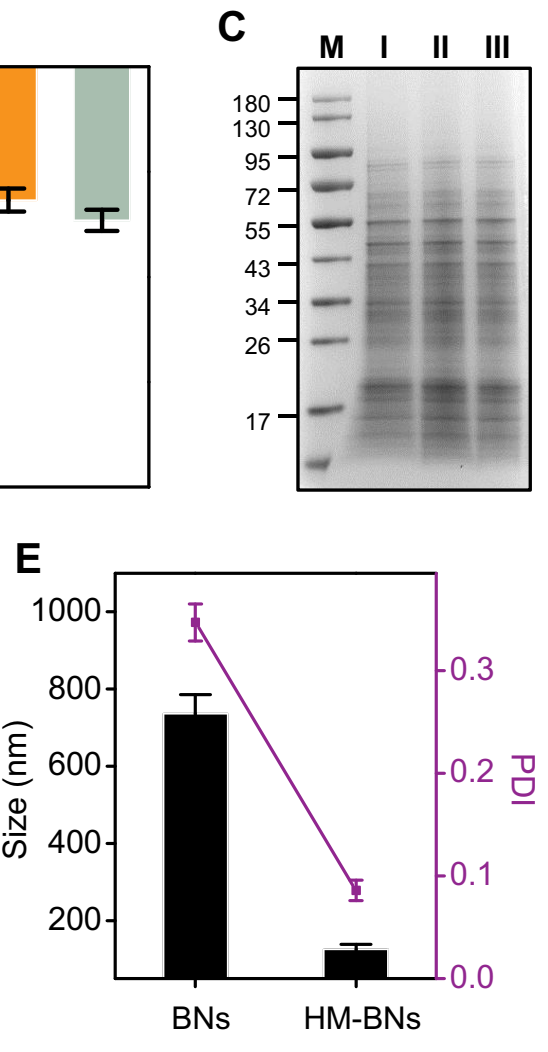

Figure 3 Characterization of HM-BNs. (A) Average diameter and (B) $\zeta$-potential of BNs, HM, and HM-BNs; (C) SDS-PAGE protein profiles of HM (I), HM after extrusion (II) and HM-BNs (III); (D) hydrodynamic diameter of BNs and HM-BNs versus time; (E) the hydrodynamic diameter and PDI of BNs and HM-BNs dispersed in PBS at I4 days. 

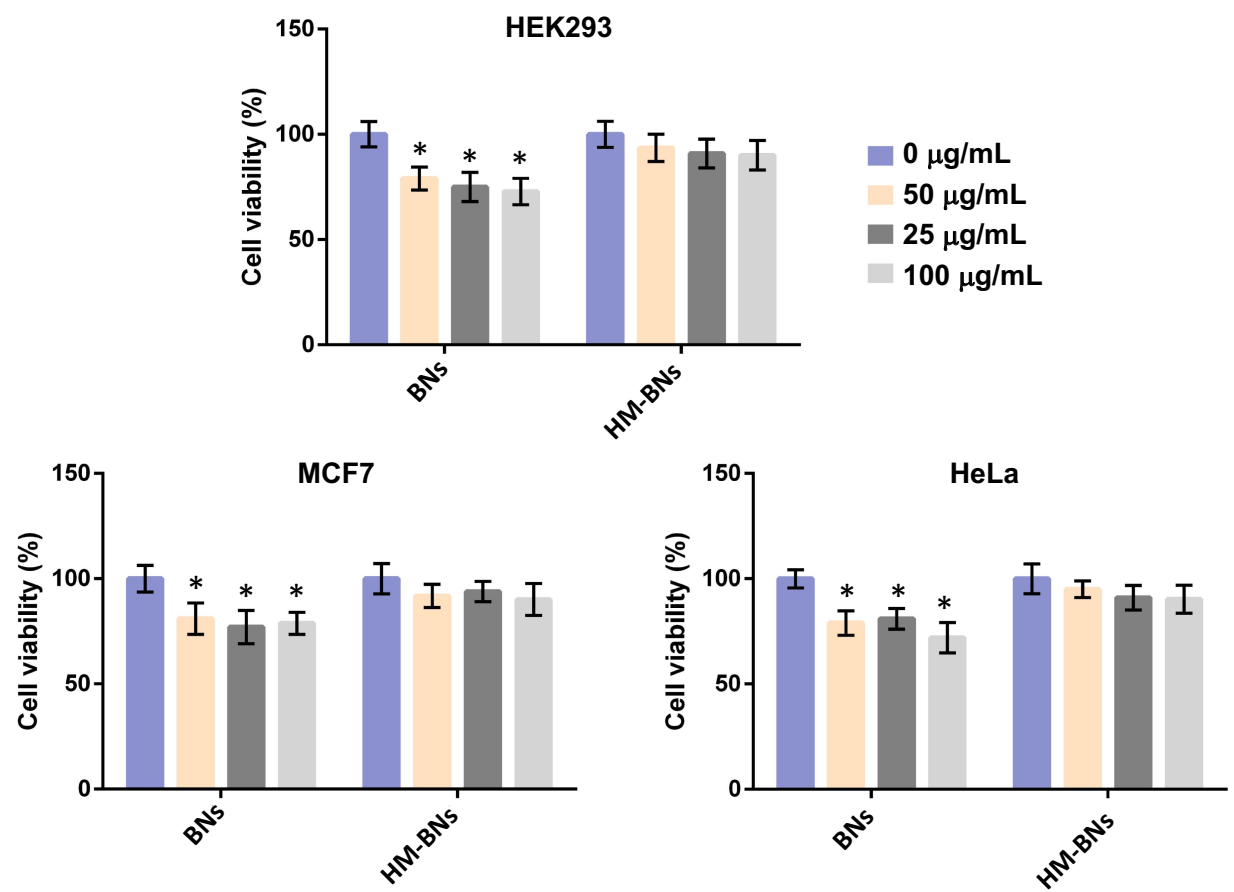

Figure 4 Cell-viability assays of HEK293, HeLa, and MCF7 cells incubated with differentconcentrations of BNs and HM-BNs for 72 hours. Data expressed as means \pm SD. $* P<0.05$.

\section{Dox Loading and In Vitro Release}

Adequate loading and efficient controlled drug release are important indicators for the evaluation of DDSs. Doxorubicin (Dox), a small-molecule chemotherapeutic drug that has effects on a variety of tumors was loaded on HM-BNs. The amount of Dox loaded on BNs was about 7 $\mu \mathrm{g} / \mathrm{mg}$, while the amount on HM-BNs was $862 \mu \mathrm{g} / \mathrm{mg}$, significantly more than BNs (Figure 5A). It was supposed that Dox was not only adsorbed in the surface of BNs but also adsorbed in cell membrane and inserted into the hydrophobic domain of the membrane bilayer, due to their lipophilic property. In addition, the mechanical extrusion also promoted loading of the drug. The in vitro drug-release behavior of Dox@HM-BNs at pH 7.4 and pH 5.0 was also studied (Figure 5B). Dox@HM-BNs showed slow release in PBS at pH 7.4 (physiological pH), and an obviously quick
A

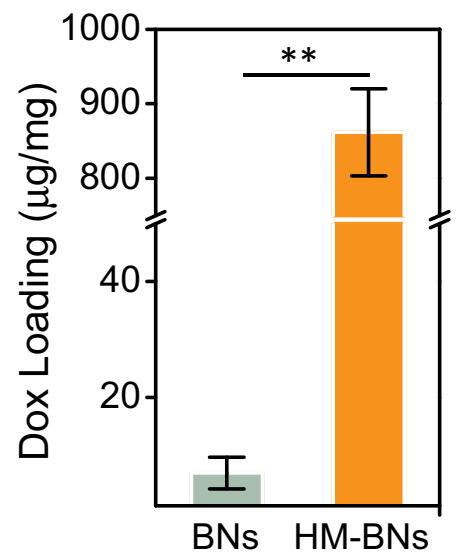

B

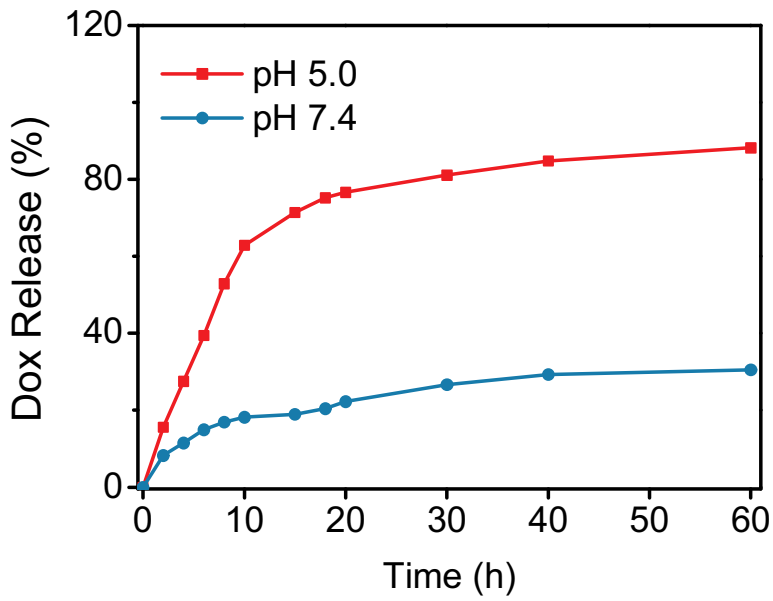

Figure 5 (A) Amount of Dox loaded on BNs and HM-BNs; (B) drug-release profiles of Dox@HM-BNs under pH 7.4 and pH 5.0. Data expressed as means \pm SD. **P<0.0I. 
release profile was observed in PBS at pH 5.0 (endosomal $\mathrm{pH}$ of cancer cells). It has been reported the structure of a cell membrane is gradually destroyed in acidic environments, and drugs attached to and enclosed in the cell membrane are thus effectively released. ${ }^{31}$ The release profiles indicated that Dox@HM-BNs were able to remain stable under physiological conditions and release drug rapidly under the acidic environment in cancer cells, which could contribute to reducing the side effects caused by unnecessary drug leakage. These results suggest enhanced drug-loading capacity and $\mathrm{pH}$-responsive controlled-release behavior of Dox@HM-BNs.

\section{Cellular Uptake of HM-BNs}

Efficient cellular uptake is crucial for the therapeutic efficacy of anticancer DDSs. Investigation of Dox@HM-BNs uptake in homologous tumor cells was performed with CLSM. The blue fluorescence indicated the nucleus and the red fluorescence from Dox indicated the distribution of drug-loaded nanoparticles in the cell. As shown in Figure 6A, HeLa cells incubated with free Dox exhibited weak red fluorescence in the nucleus, which was due to the fact that free Dox rapidly located in the nuclei of cells to bind DNA in a passive diffusion manner, and easily flushed out by Pgp. ${ }^{35}$ Furthermore, it can be observed that the Dox@HM-BN group showed significantly strong fluorescence intensity in the cytoplasm and nuclei of HeLa cells, whereas in the Dox@BN group, sporadic fluorescence appeared only in the cytoplasm of cells. These results suggested that HM modification of BNs enhanced cellular uptake by homotypic HeLa cells. Zhang et al reported on the biological functionalization of polymeric nanoparticles with a layer of membrane coating derived from cancer cells. ${ }^{31}$ They demonstrated that the coating of cancer-cell membrane on the nanoparticles facilitated their homotypic uptake by cancer cells through endocytic pathways. Flow cytometry was carried out for quantitative evaluation of the amount of Dox@HM-BNs internalized by HeLa cells (Figure 6B). In comparison with free Dox and Dox@BNs, there was remarkable enhancement of mean fluorescence intensity in cells treated with Dox@HM-BNs, further confirming improved cellular internalization of Dox@HM-BNs.

Cancer-cell membrane possesses a homologous adhesion property, which may enable HM-BNs' selective affinity for homologous tumor cells. To investigate the homotypic tumor targeting of HM-BNs, HeLa and MCF7 cells were treated with Dox@BNs and Dox@HM-BNs, respectively, then characterized with CLSM. The results are shown in Figure 7. HeLa cells treated with Dox@HM-BNs exhibited significantly higher fluorescence intensity than those treated with Dox@BNs at both incubation periods. Moreover, the red fluorescence tended to accumulate at the nuclei of HeLa
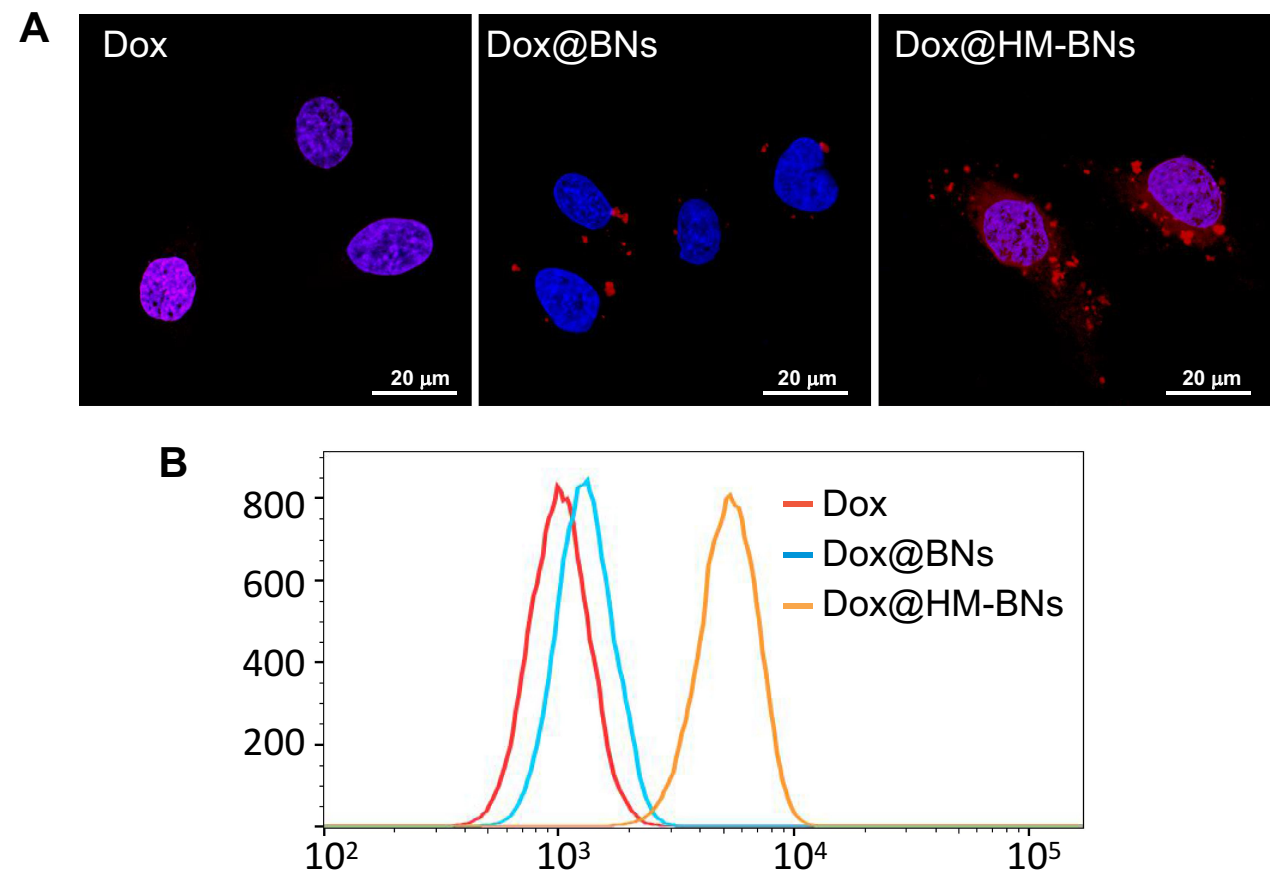

Figure 6 (A) CLSM of HeLa cells cocultured with free Dox, Dox@BNs, and Dox@HM-BNs for 4 hours (Dox, red; nucleus, blue); (B) quantitative analysis of cellular uptake by flow cytometry. 


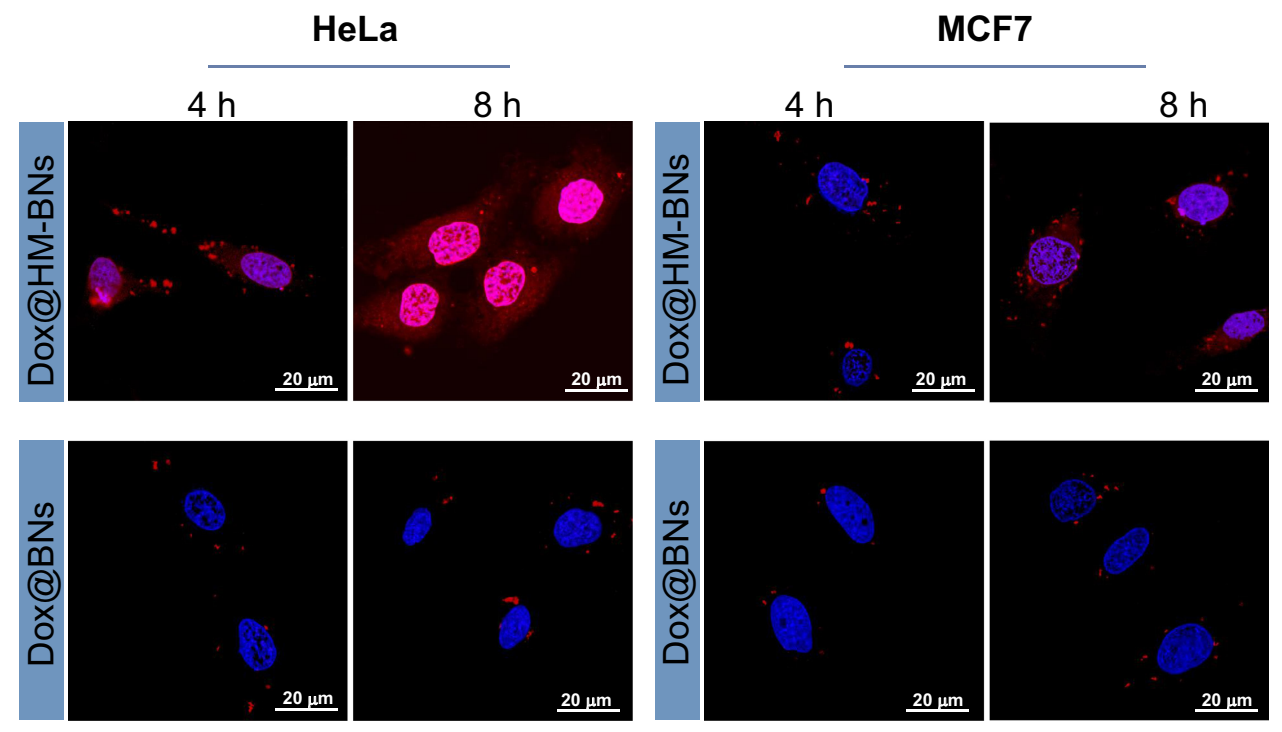

Figure 7 Cell uptake of Dox@BNs and Dox@HM-BNs in cancer cells detected under CLSM at 4 hours, and 8 hours, respectively. Nuclei were stained with DAPI.

cells after coculture with Dox@HM-BNs for 8 hours, while fluorescence localization of Dox@BNs exhibited no obvious change within the observation time, suggesting the efficient cell uptake and intracellular drug release of Dox@HM-BNs. As for MCF7 cells, little significant Dox fluorescence change was observed between Dox@BNs and Dox@HM-BNs. Cellular uptake of Dox@HM-BNs by HeLa cells and the normal cell line was also studied. Results in Figure 8A showed that the quantity of $\mathrm{B}$ atoms was four times as high in HeLa cells $(0.72 \mathrm{pg} /$ cell $)$ than in HEK293 cells $(0.18 \mathrm{pg} /$ cell), further demonstrating the selective targeting ability and enhanced cellular uptake of HM-BNs by homotypic cells. Immunoescape study of HM-BNs revealed that the internalization of HM-BNs was significantly higher than BNs by J774A.1 macrophages (Figure 8B), suggesting that encapsulation of cell membrane enabled BNs to evade recognition by the immune system to some extent. These results indicated that the biomimetic Dox@HM-BNs can effectively evade recognition by immune cells and selectively target HeLa cells by homotypic adhesive interactions of HMs.

\section{In Vitro Antitumor Effects of Dox@HN-BNs}

Encouraged by the favorable outcomes concerning selfrecognition internalization by homologous cells, the
A

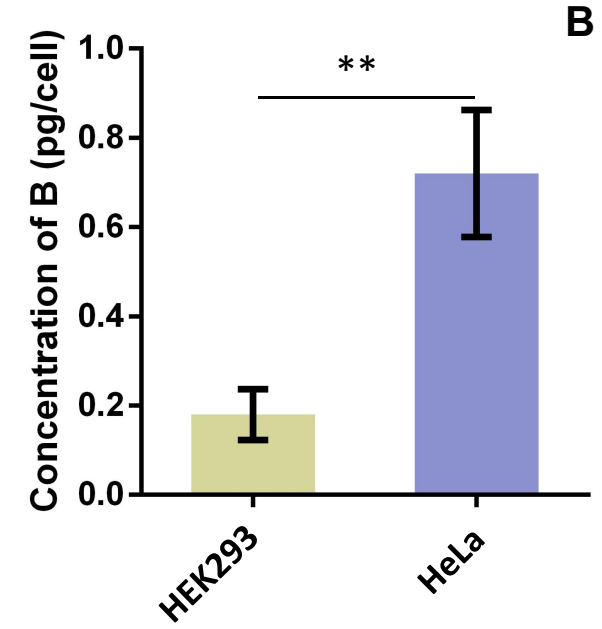

B

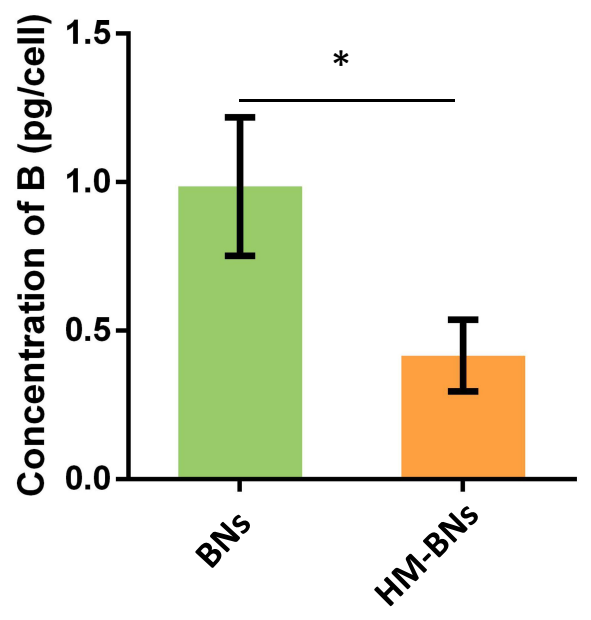

Figure 8 Quantitative analysis of intracellular concentration of boron by ICP-AES. (A) Boron in HEK293 and HeLa cells after treatment with $50 \mu$ gg/mL HM-BNs for 4 hours; (B) boron in J774A.I macrophages after treatment with BNs or HM-BNs for 4 hours. Data expressed as means $\pm S D$. $* P<0.05$; $* * P<0.01$. 


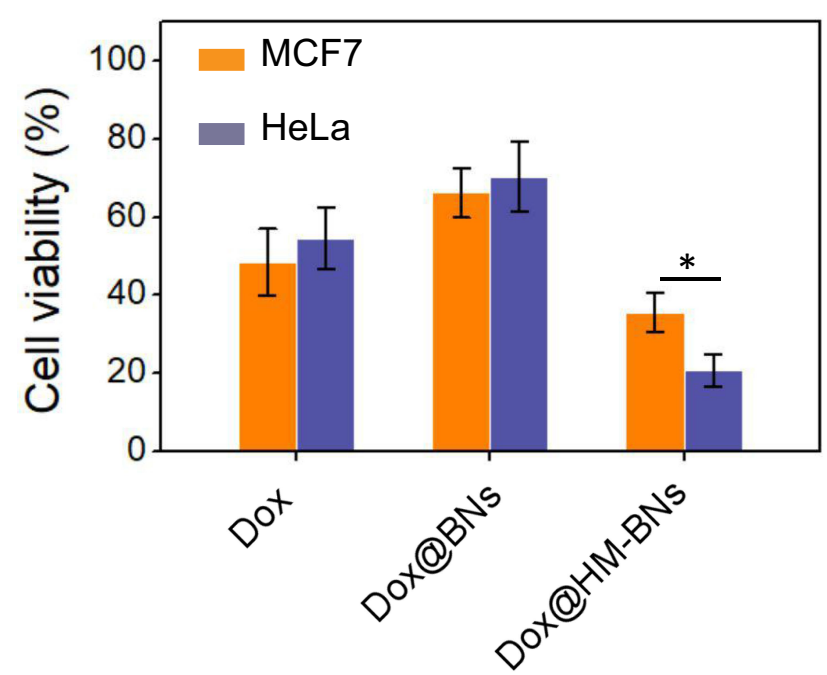

Figure 9 Viability of HeLa and MCF7 cells after coculture with free Dox, Dox@BNs, and Dox@HM-BNs on CCK8 assays. Data expressed as means \pm SD. $* P<0.05$.

therapeutic efficacy of Dox@HM-BNs on cancer cells was verified with CCK8 assays. Free Dox, Dox@BNs, and Dox@HM-BNs with the same Dox concentration were cocultured with HeLa and MCF7 cells for 24 hours. As shown in Figure 9, decreased cell viability was observed in all treatment groups. Dox@BNs showed relatively weaker lethal effects, while Dox@HM-BNs showed a significant tumor-killing effect on both cell lines. This might because the excellent solution dispersity of HM-BNs helped their uptake by cancer cells. Moreover, Dox@HM-BNs exhibited significantly higher inhibition efficacy for homotypic HeLa cells than MCF7 cells. Their enhanced killing ability in HeLa cells could be explained by the outer coating of $\mathrm{HM}$, which resulted in them being efficiently internalized by homotypic source cells.

To further confirm the anticancer efficacy of Dox@HMBNs, live/dead cell-viability assays were carried out. Green fluorescent from calcein AM indicated live cells, and red fluorescence from ethidium homodimer 1 indicated dead cells. As shown in Figure 10, Dox@HM-BN treatment in HeLa cells exhibited the strongest red fluorescence, and noticeably outperformed the other treatment groups. This result was in accordance with the CCK8 results, further confirming the satisfactory therapeutic efficacy of Dox@HN-BM. These results proved the superiority of Dox@HM-BNs for targeted cancer therapy.

\section{In Vivo Biodistribution and Tumor-Inhibition Effects of Dox@HM-BNs}

The biodistribution of HM-BNs in HeLa tumor-bearing $\mathrm{BALB} / \mathrm{c}$ nude mice was quantitatively investigated. Main organs and tumors in mice were collected 24 hours after the intravenous injection of BNs or HM-BNs with the same BN dose of $10 \mathrm{mg} / \mathrm{kg}$. The amount of boron in tumors and organs was measured by ICP-AES. As shown in Figure 11, BNs mainly existed in the liver, spleen, and lungs, indicating the hepatic excretion pathway of BNs in mice. Heterologous BNs were quickly cleared by the reticuloendothelial system when entering the living organism, resulting in low tumor accumulation. ${ }^{24}$ However, accumulation of HM-BNs at tumor sites was significantly increased in comparison to
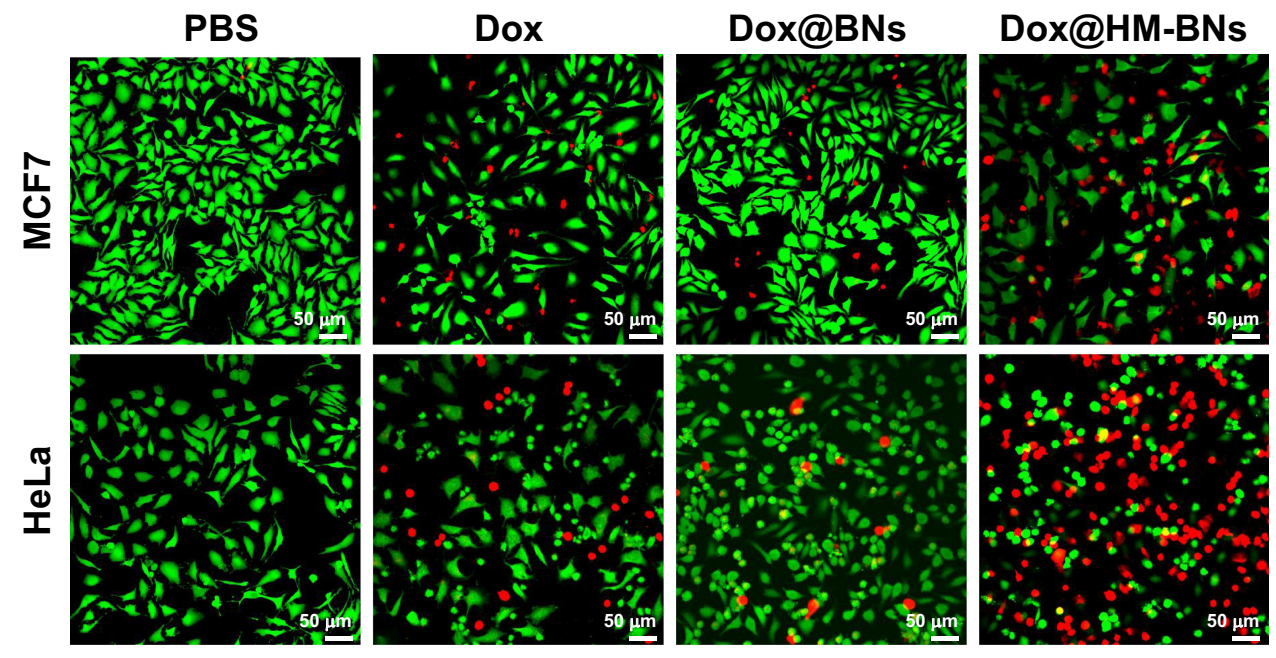

Figure 10 In vitro antitumor live/dead cell viability of cancer cells treated with PBS, free Dox, Dox@BNs, or Dox@HM-BNs at Dox concentration of $4 \mu$ g/mL (green, live cells; red, dead cells). 


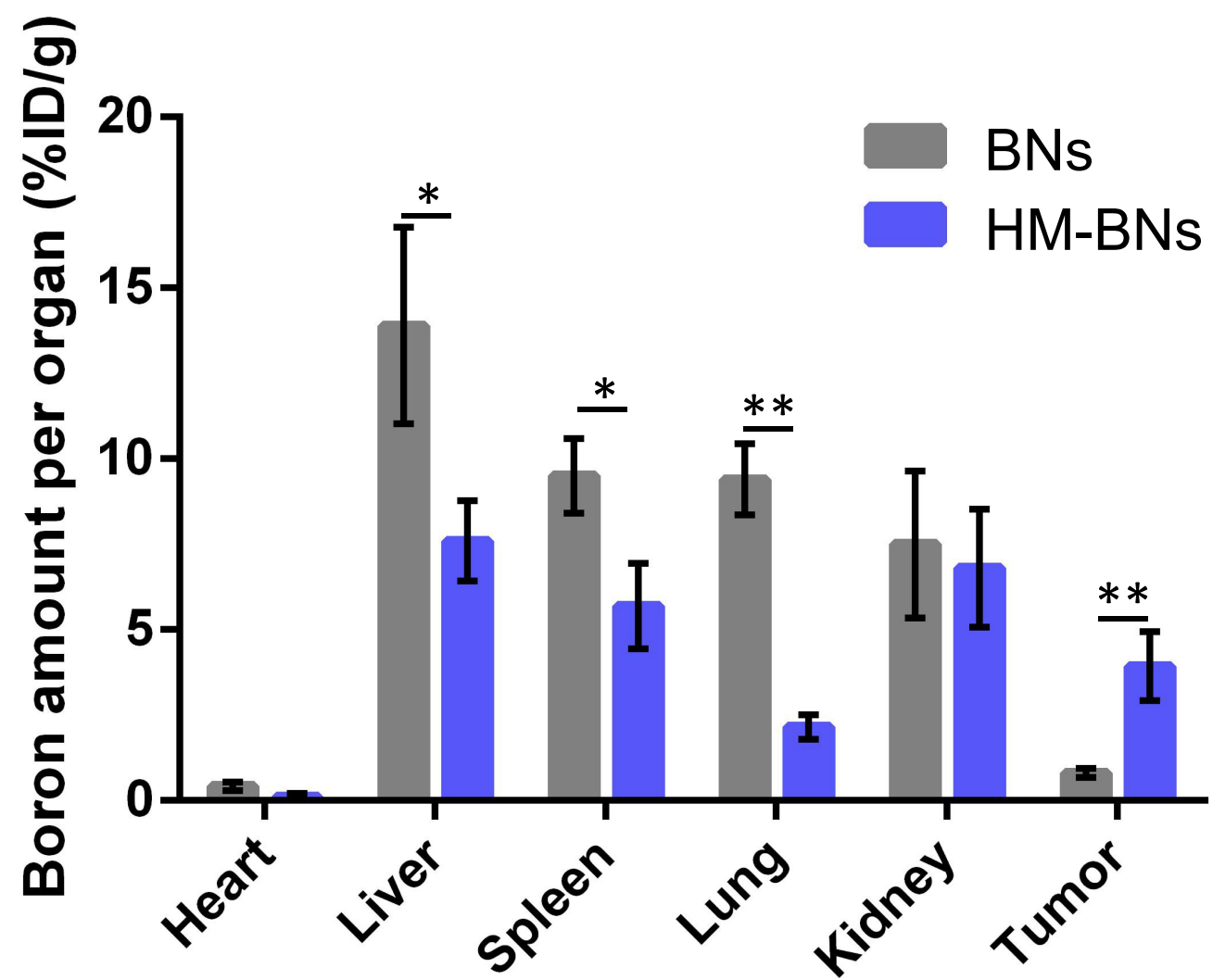

Figure I I Biodistribution of BNs and HN-BNs at 24 hours after intravenous injection $(\mathrm{n}=3)$. Data expressed as means \pm SD. $* P<0.05$; $* * P<0.0$ I.

BNs, indicating the homotypic tumor targeting of cancer cellencapsulated BNs. It was noticeable that the amount of B in the lung was decreased in HM-BNs group in comparison to BNs, which might contribute to reducing the potential toxic side effects of BNs in the lungs. The in vivo tumor-inhibition efficacy of Dox@HM-BNs was also investigated. Free Dox, Dox@BNs, or Dox@HM-BNs were systemically injected into HeLa tumor-bearing nude mice at an equivalent Dox dose of
$5 \mathrm{mg} / \mathrm{kg}$ when tumor volume had reached about $100 \mathrm{~mm}^{3}$. As illustrated in Figure 12A, tumors in the PBS-treatment group increased rapidly. Free Dox and Dox@BNs only partially delayed tumor growth, with $29.1 \%$ and $23.3 \%$ reduction in tumor volume, respectively. In contrast, significant tumor inhibition was observed in mice treated with Dox@HM-BNs, in which the tumor-inhibition rate was $75.6 \%$. This dramatically enhanced antitumor efficacy of Dox@HM-BNs could be
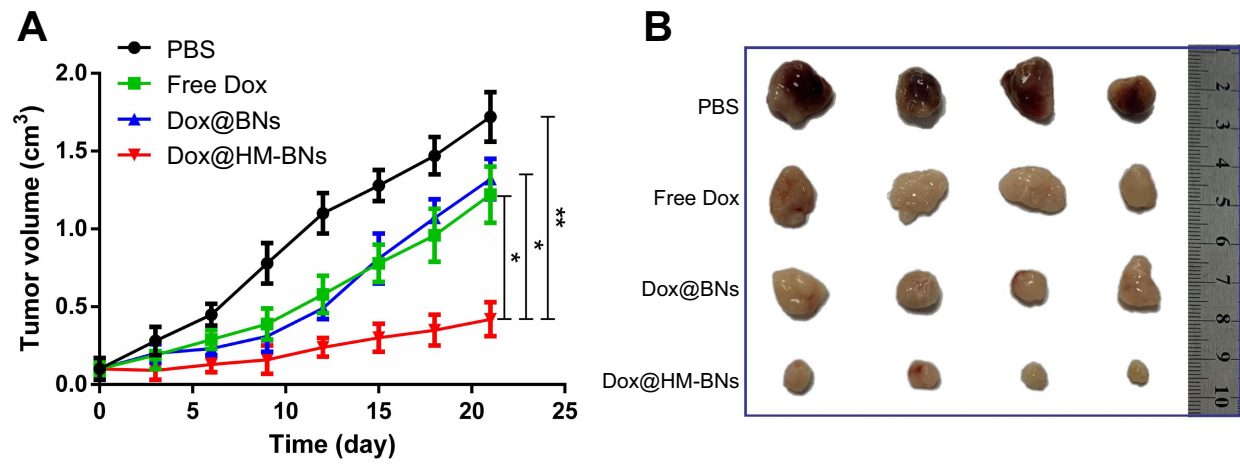

Figure 12 In vivo tumor-inhibition effect in HeLa tumor-bearing mice. (A) Tumor-volume growth after treatment with PBS, free Dox, Dox@BNs, or Dox@HM-BNs ( $\mathrm{n}=6$ ); (B) representative photographs of tumors collected from various groups of mice at the end of treatment (day $2 \mathrm{I}$ ). Data expressed as means $\pm S D$. $* P<0.05$; $* * P<0.01$. 


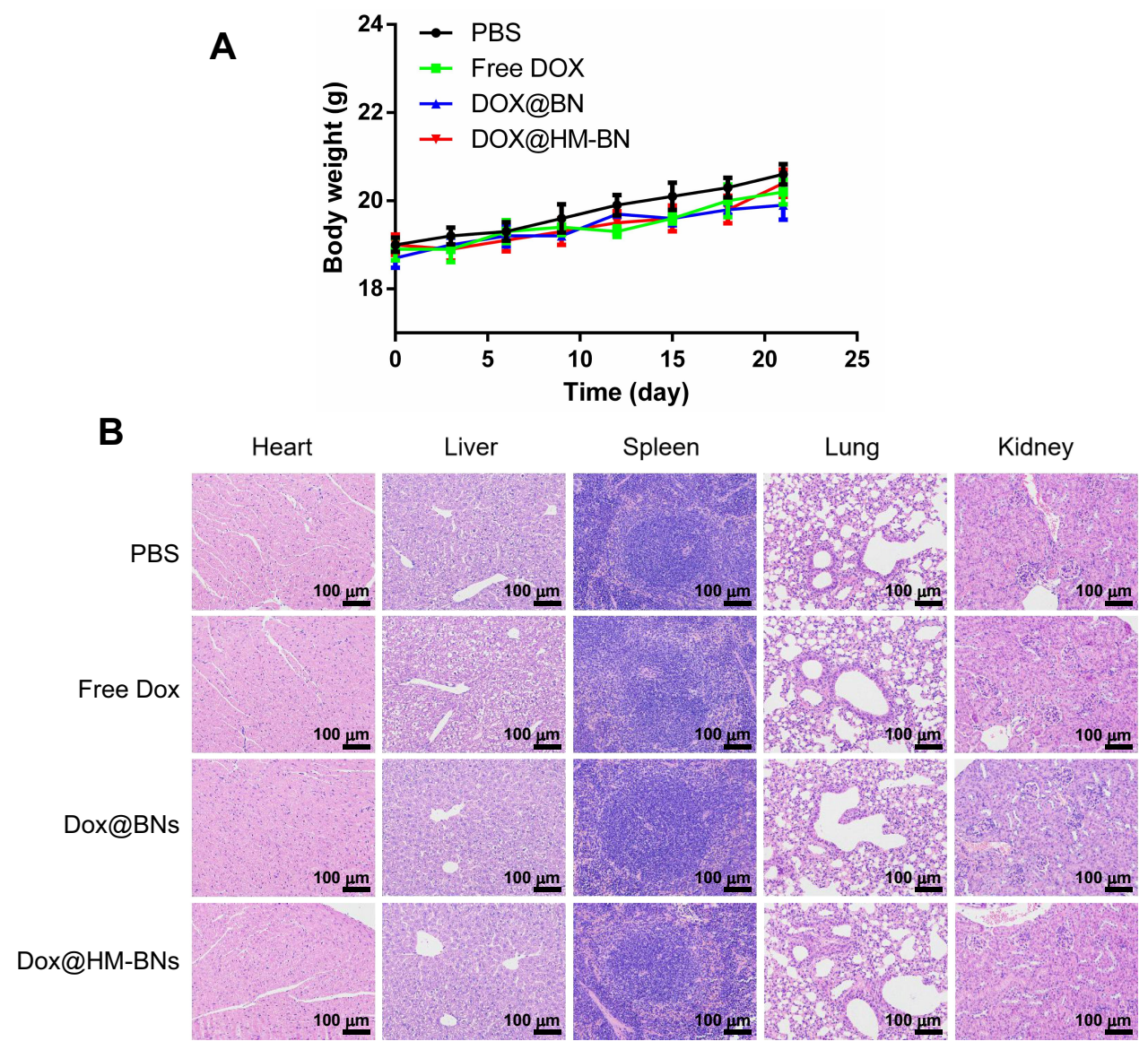

Figure 13 In vivo biosafety study. (A) Body weights of mice during therapy ( $\mathrm{n}=6$ ); (B) H\&E staining of the major organs (heart, liver, spleen, lungs, and kidneys) after different treatments.

caused by the HM coating, which could prolong their blood circulation and improve Dox accumulation at tumor site via homotypic targeting. The representative photographs of the tumors collected from various groups of mice at the end of treatment (day 21) in Figure 12B further demonstrate the enhanced tumor-inhibition efficacy of Dox@HM-BNs. Simultaneously, no noticeable body weight loss was observed in Dox@HM-BNs group (Figure 13A). At the end of treatments, major organs (heart, liver, spleen, lungs, and kidneys) were collected for the biosafety evaluation of the HM-BNbased therapeutic platform by H\&E staining. Compared with the control group, no significant tissue damage or inflamed lesions occurred in the treatment groups, suggesting the biosafety of Dox@HM-BNs (Figure 13B). Taken together, these results suggest that Dox@HM-BNs are promising as a biocompatible therapeutic nanoplatform for targeted tumor therapy.

\section{Conclusion}

A biomimetic DDS consisting of a cancer cell membranederived shell encapsulating a BN core loaded with Dox was successfully engineered in this study. Cell membrane isolated from HeLa cells was wrapped around BNs. Benefiting from the encapsulation of cell membrane, HMBNs exhibited excellent performance with regard to monodispersity, drug-loading capacity, and biocompatibility. Furthermore, the camouflaged HM composed of plentiful membrane proteins endowed the HM-BNs with homotypic targeting capacity at tumor sites. Finally, in vivo experiments also confirmed a superior tumor-inhibition effect of Dox@HM-BNs. In conclusion, this cancer-cell membrane biomimetic BN-based DDS integrates excellent dispersity, high loading capacity, and self-selective uptake by homotypic cancer cells, exhibiting strong potential for targeted tumor therapy. 


\section{Acknowledgments}

This research was funded by the National Natural Science Foundation of China (21907041, 52002239) and Natural Science Foundation of Jiangsu Province (BK20170202).

\section{Disclosure}

The authors report no conflicts of interest in this work.

\section{References}

1. Shi J, Kantoff PW, Wooster R, et al. Cancer nanomedicine: progress, challenges and opportunities. Nat Rev Cancer. 2017;17:20-37.

2. Lim EK, Kim T, Paik S, et al. Nanomaterials for theranostics: recent advances and future challenges. Chem Rev. 2015;115:327-394.

3. Barreto JA, O’Malley W, Kubeil M, et al. Nanomaterials: applications in cancer imaging and therapy. Adv Mater. 2011;23:H18-H40.

4. Zhang $\mathrm{H}$, Chen S, Zhi C, et al. Chitosan-coated boron nitride nanospheres enhance delivery of $\mathrm{CpG}$ oligodeoxynucleotides and induction of cytokines. Int J Nanomed. 2013;8:1783-1793.

5. Mukherjee S, Kotcherlakota R, Haque S, et al. Improved delivery of doxorubicin using rationally designed PEGylated platinum nanoparticles for the treatment of melanoma. Mat Sci Eng C-Mater. 2020;108:110375. doi:10.1016/j.msec.2019.110375

6. Kotcherlakota R, Srinivasan DJ, Mukherjee S, et al. Engineered fusion protein-loaded gold nanocarriers for targeted co-delivery of doxorubicin and erbB2-siRNA in human epidermal growth factor receptor-2+ovarian cancer. J Mater Chem B. 2017;5:7082-7098. doi:10.1039/C7TB01587A

7. Weber M, Lamboux C, Navarra B, et al. Boron nitride as a novel support for highly stable palladium nanocatalysts by atomic layer deposition. Nanomaterials. 2018;8(10):849. doi:10.3390/nano8100849

8. Ferreira TH, Miranda MC, Rocha Z, et al. An assessment of the potential use of BNNTs for boron neutron capture therapy. Nanomaterials. 2017;7(4):82. doi:10.3390/nano7040082

9. Emanet M, Sen O, Taskin IC, et al. Synthesis, functionalization, and bioapplications of two-dimensional boron nitride nanomaterials. Front Bioeng Biotech. 2019;7. doi:10.3389/fbioe.2019.00363

10. Zhang L, Wang X. DNA sequencing by hexagonal boron nitride nanopore: a computational study. Nanomaterials. 2016;6(6):111. doi:10.3390/nano6060111

11. Golberg D, Bando Y, Tang C, et al. Boron nitride nanotubes. Adv Mater. 2007;19:2413-2432. doi:10.1002/adma.200700179

12. Ciofani G, Raffa V, Menciassi A, et al. Folate functionalized boron nitride nanotubes and their selective uptake by glioblastoma multiforme cells: implications for their use as boron carriers in clinical boron neutron capture therapy. Nanoscale Res Lett. 2009;4:113-121. doi:10.1007/s11671-008-9210-9

13. Nakamura H, Koganei H, Miyoshi T, et al. Antitumor effect of boron nitride nanotubes in combination with thermal neutron irradiation on BNCT. Bioorg Med Chem Lett. 2015;25:172-174. doi:10.1016/j. bmcl.2014.12.005

14. Zhang H, Zhi C, Gao X. Polyethyleneimine-functionalized boron nitride nanospheres as an efficient carrier for enhancing the immunostimulatory effect of $\mathrm{CpG}$ oligodeoxynucleotides. NanomedNanotechnol. 2016;12:540. doi:10.1016/j.nano.2015.12.260

15. Zhang $\mathrm{H}$, Cheng $\mathrm{T}$, Lai L, et al. BN nanospheres functionalized with mesoporous silica for enhancing $\mathrm{CpG}$ oligodeoxynucleotide-mediated cancer immunotherapy. Nanoscale. 2018;10:14516-14524. doi:10.10 39/C8NR03820A

16. Zhang H, Yamazaki T, Zhi C, et al. Identification of a boron nitride nanosphere-binding peptide for the intracellular delivery of $\mathrm{CpG}$ oligodeoxynucleotides. Nanoscale. 2012;4:6343-6350. doi:10.1039/ c2nr31189e
17. Zhi C, Meng W, Yamazaki T, et al. BN nanospheres as CpG ODN carriers for activation of toll-like receptor 9. J Mater Chem. 2011;21:5219-5222. doi:10.1039/c1jm10199d

18. Feng S, Zhang $\mathrm{H}$, Zhi $\mathrm{C}$, et al. $\mathrm{pH}$-responsive charge-reversal polymer-functionalized boron nitride nanospheres for intracellular doxorubicin delivery. Int $J$ Nanomed. 2018;13:641-652. doi:10.21 47/IJN.S153476

19. Feng S, Zhang H, Yan T, et al. Folate-conjugated boron nitride nanospheres for targeted delivery of anticancer drugs. Int J Nanomed. 2016;11:4573-4582. doi:10.2147/IJN.S110689

20. Feng S, Zhang $\mathrm{H}, \mathrm{Xu} \mathrm{S}$, et al. Folate-conjugated, mesoporous silica functionalized boron nitride nanospheres for targeted delivery of doxorubicin. Mat Sci Eng C-Mater. 2019;96:552-560. doi:10.1016/ j.msec.2018.11.063

21. Yin J, Li J, Hang Y, et al. Boron nitride nanostructures: fabrication, functionalization and applications. Small. 2016;12:2942-2968.

22. Hu C-MJ, Fang RH, Zhang L. Erythrocyte-inspired delivery systems. Adv Healthc Mater. 2012;1:537-547. doi:10.1002/adhm.201200138

23. Gao W, Hu C-MJ, Fang RH, et al. Surface functionalization of gold nanoparticles with red blood cell membranes. Adv Mater. 2013;25:3549-3553. doi:10.1002/adma.201300638

24. Feng S, Li H, Ren Y, et al. RBC membrane camouflaged boron nitride nanospheres for enhanced biocompatible performance. Colloids Surf B. 2020;190:110964. doi:10.1016/j.colsurfb.2020. 110964

25. Ku SH, Kim K, Choi K, et al. Tumor-targeting multifunctional nanoparticles for siRNA delivery: recent advances in cancer therapy. Adv Healthc Mater. 2014;3:1182-1193. doi:10.1002/adhm. 201300607

26. Sakurai Y, Kajimoto K, Hatakeyama H, et al. Advances in an active and passive targeting to tumor and adipose tissues. Expert Opin Drug Del. 2015;12:41-52. doi:10.1517/17425247.2015.955847

27. Fang RH, Kroll AV, Gao W, et al. Cell membrane coating nanotechnology. Adv Mater. 2018;30(23):1706759. doi:10.1002/ adma.201706759

28. Deng G, Sun Z, Li S, et al. Cell-membrane immunotherapy based on natural killer cell membrane coated nanoparticles for the effective inhibition of primary and abscopal tumor growth. ACS Nano. 2018;12:12096-12108. doi:10.1021/acsnano.8b05292

29. Ouyang X, Wang X, Kraatz HB, et al. A Trojan horse biomimetic delivery strategy using mesenchymal stem cells for PDT/PTT therapy against lung melanoma metastasis. Biomater Sci. 2020;8:1160-1170. doi:10.1039/C9BM01401B

30. Wang $\mathrm{H}$, Bremner $\mathrm{DH}, \mathrm{Wu} \mathrm{K}$, et al. Platelet membrane biomimetic bufalin-loaded hollow $\mathrm{MnO}_{2}$ nanoparticles for MRI-guided chemo-chemodynamic combined therapy of cancer. Chem Eng J. 2020;382:122848. doi:10.1016/j.cej.2019.122848

31. Zhu JY, Zheng DW, Zhang MK, et al. Preferential cancer cell self-recognition and tumor self-targeting by coating nanoparticles with homotypic cancer cell membranes. Nano Lett. 2016;16: 5895-5901. doi:10.1021/acs.nanolett.6b02786

32. Tang C, Bando Y, Huang Y, et al. Synthetic routes and formation mechanisms of spherical boron nitride nanoparticles. Adv Funct Mater. 2008;18:3653-3661. doi:10.1002/adfm.200800493

33. Chai Z, Ran D, Lu L, et al. Ligand-modified cell membrane enables the targeted delivery of drug nanocrystals to glioma. ACS Nano. 2019;13:5591-5601. doi:10.1021/acsnano.9b00661

34. Jiang Y, Krishnan N, Zhou J, et al. Engineered cell-membrane-coated nanoparticles directly present tumor antigens to promote anticancer immunity. Adv Mater. 2020;32(30):2001808. doi:10.1002/adma.202 001808

35. Sui J, He M, Yang Y, et al. Reversing P-glycoprotein-associated multidrug resistance of breast cancer by targeted acid-cleavable polysaccharide nanoparticles with lapatinib sensitization. ACS Appl Mater Inter. 2020;12:51198-51211. doi:10.1021/acsami.0c13986 


\section{Publish your work in this journal}

The International Journal of Nanomedicine is an international, peerreviewed journal focusing on the application of nanotechnology in diagnostics, therapeutics, and drug delivery systems throughout the biomedical field. This journal is indexed on PubMed Central, MedLine, CAS, SciSearch ${ }^{\mathbb{B}}$, Current Contents ${ }^{\mathbb{B}} /$ Clinical Medicine,
Journal Citation Reports/Science Edition, EMBase, Scopus and the Elsevier Bibliographic databases. The manuscript management system is completely online and includes a very quick and fair peer-review system, which is all easy to use. Visit http://www.dovepress.com/ testimonials.php to read real quotes from published authors. 